\title{
LEACH Algorithm Improvement Based on Cluster Head Selection
}

\author{
Min-Sheng Tan, Chen-Cheng Wang, Miao Guo, Ting Xiang, Qun-Zhong Peng, Yang-Wei Li \\ School of Computer Science and Technology University of South China Hengyang, Hunan, 421001, China \\ E-mail: tanminsheng65@163.com,wcc93@qq.com,1079516278@qq.com, 15096025554@163.com,358996619@qq.com, \\ 574618939@qq.com
}

\begin{abstract}
In order to solve the problem of LEACH Algorithm cannot guarantee the larger residual energy node absolutely were elected as cluster head, and it consumes large amounts of energy for double counting of cluster head election, at the same time, the influence of the node distance for cluster head election is not included. The improved LEACH algorithm has solved the problems very well, it makes a use of cluster heads projection to decrease the number of cluster competition by combining with the optimal cluster head selection algorithm, which can reduce the node energy consumption, then to achieve equilibrium network energy consumption and prolong the survival time of the network. And the experimental results are shown that the improved LEACH algorithm balanced the energy consumption of the entire network, significantly reduced the power consumption of the nodes in the monitoring area, delayed the first dead nodes appear on the number of rounds, and prolonged the life cycle of monitoring network.
\end{abstract}

Keywords-the improvement of LEACH algorithm; optimal cluster head selection; cluster heads forecasting mechanism; time schedule

\section{INTRODUCTION}

LEACH Algorithm[1][2] is a typical node-based clustering of adaptive clustering topology algorithm. The cluster idea present in [3] has a significant reflection to the research of $\mathrm{WSN}$ (wireless sensor network) routing protocols. LEACH has hierarchical topology control structure and energy-saving features to round as a cycle, each cycle can be divided into node initialization phase, the cluster head selection phase, cluster formation phase and data stability transfer phase[4].LEACH algorithm uses a loop for each selected cluster head node for regional monitoring to make not yet served as the cluster head node promptly to sleep in monitoring area, reducing the energy consumption[5] of most nodes, thus balancing the energy of the entire monitoring network consumption, so that increases the lifetime of the monitoring network[6]. However, the cluster head node has responsibility to real-time monitoring area changes, receiving instructions passed by sink nodes and message sent by ordinary nodes (non-cluster head node), and passing the collected data through a simple packing process to the sink node, therefore, the energy consumption of cluster head node a lot more than ordinary nodes. And the implementation of LEACH Algorithm needs two essential premises Firstly,ensure that all nodes in the network have the same energy magnitude in the initialization. Secondly,in any round cluster head nodes energy consumption are the same, which can make the whole network nodes tend to balance energy consumption, thereby extending the network lifetime.
However, the actual situation is not so idealistic, firstly node energy cannot be guaranteed the same; Second, as the nodes randomly distributed at different distances that will result in communication energy consumption are different; Finally the cluster head node production is only decided by threshold $T(\mathrm{n})$, and the value of $T(\mathrm{n})$ is related to two factors: the percentage $(P)$ of cluster head nodes in the whole network and implementing rounds $(r)$ of cluster head election, but the size of $P$ is related to network coverage and density, and it is difficult to get the value of. Thus, LEACH algorithm has the following disadvantages:

- Dump energy of nodes is not taken into account in cluster head node election.

- Repeat calculation is irrational in cluster head election.

- Irrational distribution of cluster head node.

- Cluster head selection did not consider the data communication distance.

This paper proposes an new improved LEACH algorithm, it has solved the above problem very well. Considering both the influence of the residual energy and the distance to the cluster head nodes' selection, this paper introduces a better selection of the optimal cluster head and builds a cluster head prediction mechanism which make the node, with more residual energy and short distance, big probability elected of cluster heads to avoid early death and prolong the lifetime of the network [7-9].

\section{THE IMPROVED LEACH ALGORITHM}

The selection of cluster head affects the death time of the nodes in the network and the network s lifetime, so it becomes the key to the problem of how to choose the more residual energy and location optimal nodes. Although the LEAGH algorithm and other improvement algorithm[10][11] have dealt with the problem of node randomly as cluster heads, which in theory can satisfy each node has 100 percent chance as a cluster head. But in practice, LEACH algorithm does not take into account the residual energy of nodes and node communication distance, which can lead to nodes premature death due to the energy premature consumption, affecting the performance of the network.

\section{A. The Selection of the Optimal Cluster Head}

Considering the influence of the residual energy and distance to the cluster head nodes selection, the improved threshold function $\mathrm{T}(\mathrm{n})$ is introduced in this paper, it $\mathrm{s}$ defined as equation (1): 


$$
T(n)=\left\{\begin{array}{lr}
\frac{p}{1-p *[r \bmod (1 / p)]} * \rho, n \in G \\
0, & \text { other }
\end{array}\right.
$$

In (1), $\mathrm{P}$ is the ratio of elected cluster heads number in all nodes; $r$ is the number of rounds in the current cluster head election cycle ; $\mathrm{r} \bmod (1 / \mathrm{P})$ is the number of cluster head in this selection cycle of cluster head; $G$ is the collection of nodes that has not yet elected cluster head in this round of cluster head election; is a dynamic variable of residual energy of nodes in the network, it $\mathrm{s}$ defined as equation (2):

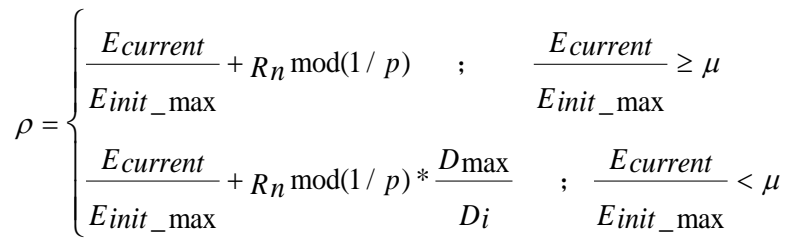

$E_{\text {init_max }}$ is the biggest energy of nodes before the network initialization; $E_{\text {current }}$ is the current node's residual energy; $R_{n}$ is the number of not elected cluster head node s rounds, whose initial value (first run for cluster head nodes) is 0 , after each time of the node take part in an election and is not elected cluster head, the value of $R_{n}$ would automatically add 1, while elected the value of $R_{n}$ would return to 0 ; $D_{\max }$ is the farthest distance between any nodes in this cluster with cluster head nodes; $D_{i}$ is the current node to the cluster head node straight-line transmission distance.

In (2), through a large number of experiments, the best value of time threshold is 0.05 .

\section{B. The Build of Cluster Head Prediction Mechanism Algorithm}

In the control of clustered topology, requires switching a lot of control packet to competition cluster heads during the cycle of each cluster head election, resulting in wasted a lot of energy. The nodes with smaller residual energy are likely to die prematurely, which would affect the network's survival. Therefore, combine with the optimal cluster head selection algorithm to predict cluster heads and reduce the node energy consumption by decreasing the number of cluster competition, then to achieve balanced energy consumption of the network and prolong the survival time of the network.

1) Construction of cluster head prediction mechanism. Definition 1 The $\mathrm{r}$ represents the performed rounds number of current network, $\mathrm{n}$ is the number of cluster head nodes after $r$ rounds of cluster head prediction, and when the mod (r, $\mathrm{n})=0$, it means the current cluster heads need to perform the next round of cluster head prediction algorithm.

Definition 2 Based on the number of the nodes in the cluster, build a time scheduling table TMDA of cluster head nodes to take turns to use active node in scheduling table intensively.
At the same time to build a three time parameter, Ta, Tb, Tc. Among them, the $\mathrm{Ta}>=$ (frame size / channel speed), $\mathrm{Tb}=\mathrm{Ta} *($ nodes number 1$)$, and $\mathrm{Tr}$ is predetermined round time length;

Definition 3 The $\mathrm{R}=\{\mathrm{r} 0, \mathrm{r} 1,, \mathrm{rn}\}$ and $\bmod (\mathrm{ri}, \mathrm{n})=0,0 \mathrm{i}$. When $\mathrm{r} \mathrm{R}$ cluster nodes perform a cluster head prediction algorithm, calculate the next $\mathrm{n}$ cluster nodes and set the calculated nodes in TDMA(Time Division Multiple Address) scheduling table. $\mathrm{Sy}=\{\mathrm{CYi} \mid 0 \mathrm{i}\}, \mathrm{CY} 0$ is the first cluster head node.

Definition 4 The E=NULL expresses the collection of cluster node's residual energy, $\mathrm{n} 2$ is the number of cluster nodes, $\mathrm{n} 1$ is the number of non-cluster nodes. And $\mathrm{Sn}=\{\mathrm{CNi} \mid 1=<\mathrm{i}=<\mathrm{n} 1\}$ is the collection of non-cluster nodes. Cluster heads prediction algorithm can be realized through the following steps:

Step 1: From the collection $\mathrm{CNi}$ of non-cluster-head to remove a node $\mathrm{Sn}$, the initial energy denoted Einit

Step 2:Take a cluster head node CYi from the cluster head set and calculate the size of the energy factor of the node $\mathrm{CNi}$ and node $\mathrm{CYi}$ according to (2). Then traverse each node and record the amount of residual energy.

Step 3:Detect whether nodes in the cluster head set have been traversed totally. If all nodes have been already traversed, put the node CNi into set $\mathrm{E}$ and then go to step 4. Otherwise, the second step will be to continue to execute until the cluster head node traversed to the last.

Step 4:Detect whether nodes in the non cluster head set Sn have been traversed totally. If already traversed all nodes, proceed to step 5.Otherwise, go back to step 1 and continue to traverse the nodes in set Sn.

Step 5:Find the node Emax with the largest residual energy from the set $\mathrm{E}$, add the corresponding node CYmax as a new prediction cluster head to the set Sy, at the same time, remove the node from the set $\mathrm{Sn}$, and set $\mathrm{E}=$ NULL.

Step 6:Determine whether there are $n+1$ nodes in the set Sy. If so, the node will be written to the TDMA schedule table, or return to step 1 to continue the prediction process.

Therefore, there are n predictor cluster heads in Sy at last, and write all of them to TDMA schedule table in accordance with the size of the residual energy. In each round, node as a cluster head in turn based on time fragment size, the consumption of cluster head will be balanced to each predict cluster heads.

The cluster head node added to dispatch table after a cluster head forecasts, whose energy consumption mathematical formula is as equation (3):

$$
E_{\text {total }}= \begin{cases}L_{d} E_{\text {elec }}+L_{d} \varepsilon_{f s} d_{i}^{2}+2 L_{c} E_{\text {elec }}+L_{c} \varepsilon_{f s} d_{i}^{2}, & d_{i}<d_{0} \\ L_{d} E_{\text {elec }}+L_{d} \varepsilon_{m p} d_{i}^{4}+2 L_{c} E_{\text {elec }}+L_{c} \varepsilon_{m p} d_{i}^{4}, & d_{i} \geq d_{0}\end{cases}
$$

In (3), is the total of energy consumption of the cluster head note, is the transmit data packet size; is a data control packet size; is transmitted energy consumption for 1bit data; is a free power factor; is multipath fading power factor; is transmission distance; is transmission distance threshold.

Construction of the time slice scheduling 


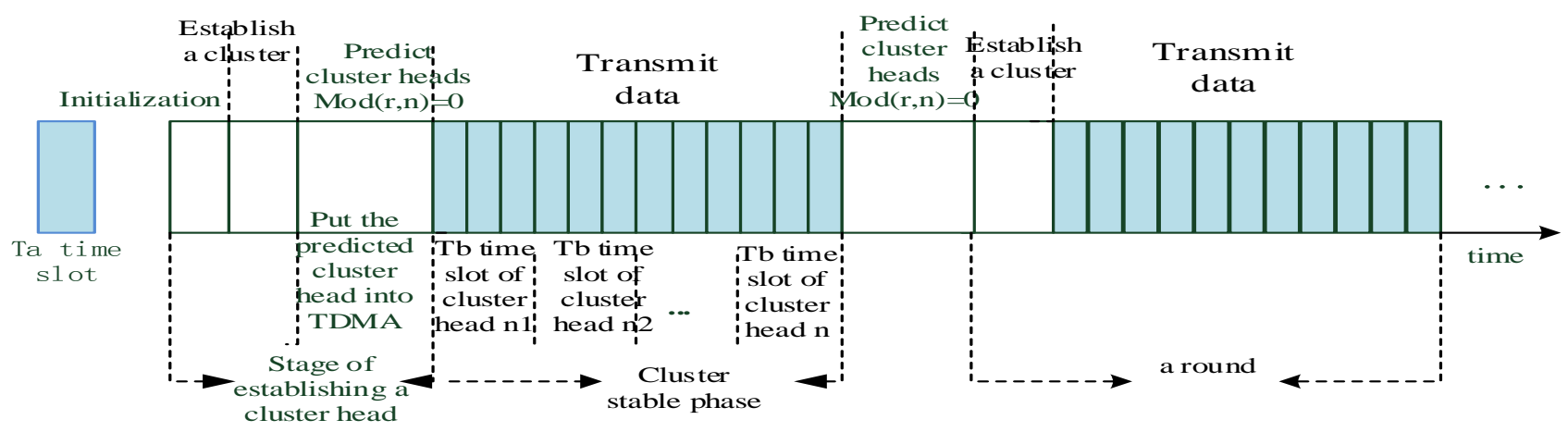

Figure 1. Time slice clustering based on the mechanism of cluster head prediction.

Fig.1 is a graph of time slice clustering based on the mechanism of cluster head prediction. It shows that the $n$ nodes are placed to the TDMA schedule on the base of the size of the residual energy after a cluster head prediction mechanism. After the network into clusters, the number of node is $\mathrm{n}$ of current network, first determine the round length $\mathrm{Tr}$ and slot length $\mathrm{Ta}$, then $\mathrm{Tb}=\mathrm{N}-1 * \mathrm{Ta}$. Therefore, has $\mathrm{k}=$ $\mathrm{Tr} / \mathrm{Tb}$ times as cluster head. When the algorithm is executed, time slot Ta will no longer be allocated, but at $\mathrm{Tb}$ time slots the $\mathrm{n}$ prediction node in the dispatch table to rotate as cluster heads. At the same time, each node can only transmit data in Ta time slot. Prediction of cluster will become a common node after $\mathrm{K}$ times as cluster head node, then the new node as the next one to finish the work of Tb time slot. Each new cluster head transition probability is $\mathrm{P}=1 / \mathrm{R}, \mathrm{R}$ represents not elected cluster head node number, $\mathrm{R}$ may be included in the communication frame between the nodes. After cluster data into the stable phase, assuming that the node energy consumption in the time slot $\mathrm{Tb}$ is $\mathrm{Eb}, \mathrm{n}$ predict nodes energy consumption in LECAH algorithm is $\mathrm{n} * \mathrm{~Eb}$, however, in this algorithm, $\mathrm{n}$ predict nodes energy consumption is $(1+n / N) * E b$, other $(N-n)$ nodes each ordinary node energy only $(\mathrm{n} / \mathrm{N}+1) * \mathrm{~Eb}$. Therefore, The total energy consumption of the predict cluster heads must be less than $\mathrm{n} * \mathrm{~Eb}$, the ordinary node energy consumption less than $\mathrm{Eb}$. Therefore, the algorithm almost split energy on each node of each cluster head to avoid excessive forward data and information gathering as much energy consumption, early entry into premature death, but also reduces the energy consumption of ordinary nodes, the entire network can reach equilibrium measure of consumption and prolong the network lifetime.

\section{The Improved LEACH Algorithm Flow Chart}

Adding cluster head prediction mechanism and time schedule to improve the shortage of cluster head selection of LEACH algorithm, the improved LEACH algorithm has added prediction of cluster to the TDMA schedule to avoid the energy consumption of computing the entire network because of excessive cluster selection, helped to protect the nodes whose remaining energy are relatively less, prevented the nodes to enter into the dead period too early, and reduced calculation times for the whole network energy consumption balance. The improved process of the cluster head prediction mechanism based on LEACH algorithm shown in Figure. 2

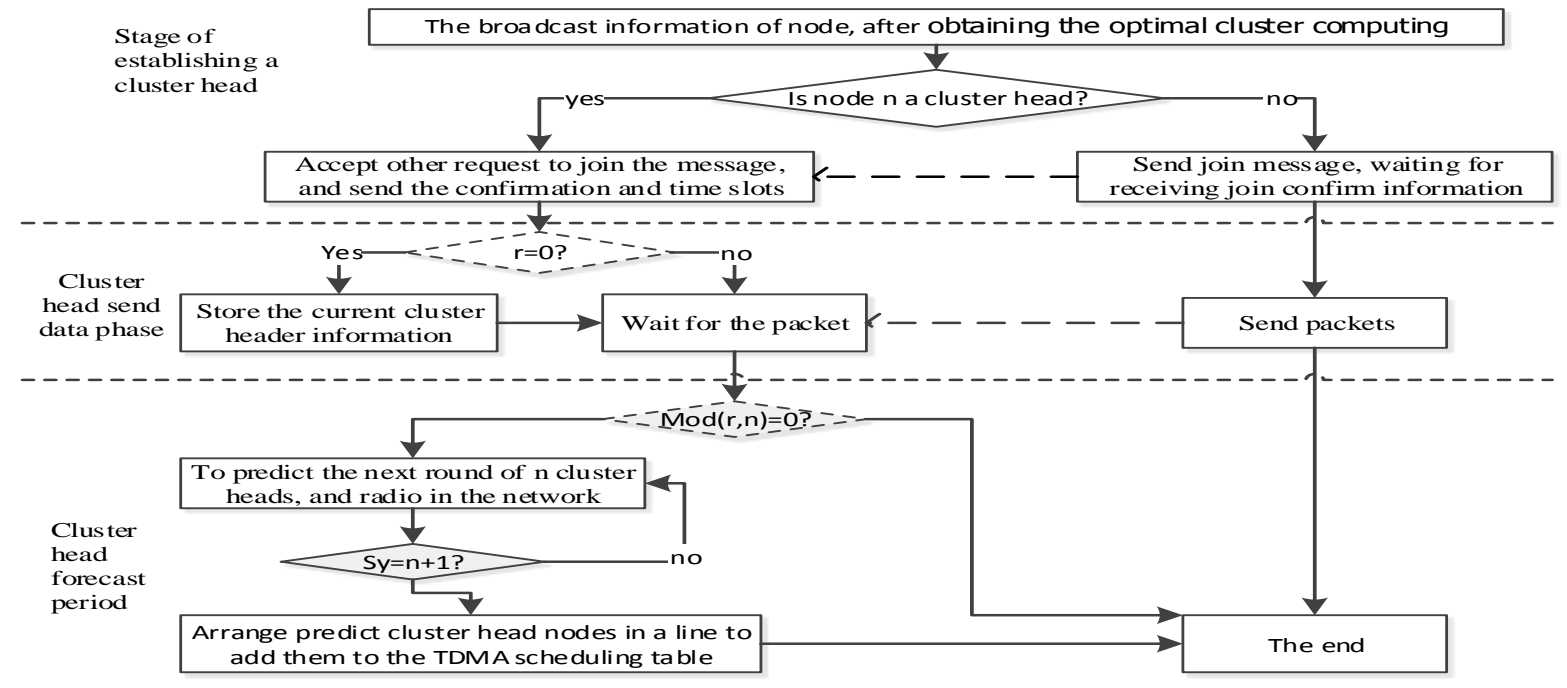

Figure 2. The improved leach algorithm flow chart of prediction mechanis 


\section{THE SIMULATION ANALYSIS}

\section{A. The summary of Experimental Platform}

TinyOS system is specifically designed for wireless sensor network node, component-oriented and event-driven embedded operating system. It also has four technology advantages: component-based programming, event-driven mechanism, lightweight threads technology, two layer of scheduling mode and proactive messaging .

TOSSIM is the simulator of TinyOS[12], which provides hardware external interfaces for software simulation, allowing the same code of the actual node machine to perform large-scale nodes simulation in general computers, to capture the network behavior and interactions from thousands of nodes. The users can add the functions needed into TOSSIM, replace hardware features with module components to perform simulation. It will be very convenient, flexible, fast and accurate.

\section{B. The Improved LEACH Algorithm S Simulation And Analysis}

1) Emulation scene and parameter settings

The simulation using TOSSIM simulation platform, the concrete parameter settings is shown in Table1 .

TABLE I. THE CONCRETE PARAMETER SETTINGS

\begin{tabular}{|c|c|}
\hline parameter name & value \\
\hline Network size & $100 \mathrm{~m} * 100 \mathrm{~m}$ \\
\hline Base station location & 50,50 \\
\hline Number of nodes & 100 \\
\hline Initial energy & $0.5 \mathrm{~J} 0.75 \mathrm{~J}$ \\
\hline $\begin{array}{l}\text { Energy consumption of } \\
\text { wireless transceiver }\end{array}$ & $50 \mathrm{~nJ} / \mathrm{bit}$ \\
\hline $\begin{array}{l}\text { Amplifier gain energy } \\
\text { consumption }\end{array}$ & $100 \mathrm{pJ} / \mathrm{bit} / \mathrm{m}^{2}$ \\
\hline Data frame size & 300Bytes \\
\hline Broadcast the frame size & 30Bytes \\
\hline The threshold distance & $275 \mathrm{~m}$ \\
\hline Round of the time & $15 \mathrm{~s}$ \\
\hline time slot & $3.2 \mathrm{~ms}$ \\
\hline $\begin{array}{l}\text { Data fusion energy } \\
\text { consumption }\end{array}$ & $50 \mathrm{~nJ}$ \\
\hline
\end{tabular}

\section{2) Emulation results and analysis}

a) The death trend of nodes in LEACH and The improved LEACH algorithm

Experimental simulation 100 nodes, the main statistical the algorithms of LEACH algorithm and the improved LEACH algorithm's dead nodes, such as listed in Table 2.

Comparison of the data from the Table II it can be seen that the improved LEACH algorithm delayed the first dead node than the LEACH algorithm. In order to analyze the death trend of nodes under the two algorithms, we converted the table into graph, as the Figure.3.

TABLE II. THE LEACH ALGORITHM AND THE IMPROVED LEACH ALGORITHM'S DEAD NODES

\begin{tabular}{|c|c|c|}
\hline $\begin{array}{c}\text { The number of } \\
\text { dead nodes }\end{array}$ & $\begin{array}{c}\text { LEACH simulation } \\
\text { rounds }\end{array}$ & $\begin{array}{c}\text { Improved LEACH } \\
\text { simulation rounds }\end{array}$ \\
\hline 1 & 881 & 1094 \\
\hline 2 & 1016 & 1127 \\
\hline 3 & 1034 & 1138 \\
\hline 4 & 1041 & 1227 \\
\hline 5 & 1045 & 1384 \\
\hline 6 & 1055 & 1395 \\
\hline 7 & 1160 & 1410 \\
\hline 8 & 1211 & 1467 \\
\hline 9 & 1230 & 1485 \\
\hline 10 & 1237 & 1510 \\
\hline
\end{tabular}

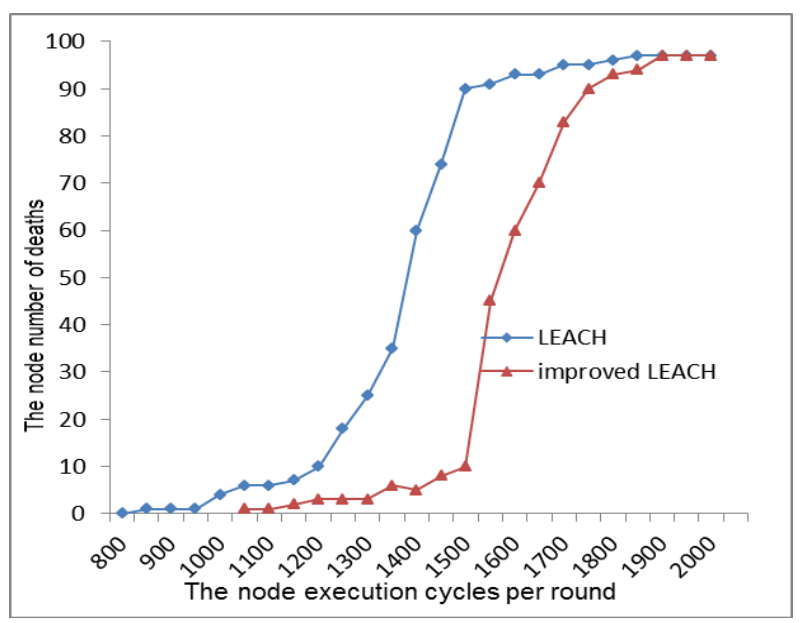

Figure 3. Dead nodes rising trend of the LEACH algorithm and the improved LEACH algorithm 
The first death node of improved LEACH algorithm occurs in the round 1094, rising $24.2 \%$ and $10.1 \%$ compared to $\mathrm{LEACH}$. And the death node rate of improved LEACH algorithm rises slowly before 1500 rounds, less than $10 \%$ death node, however, when it comes to LEACH, death node rate rise rapidly after 1200 rounds, and the rate almost over $70 \%$ to 1500 rounds. Therefore, the simulation results show that: improved LEACH algorithm balances the energy of the

entire network and guarantee the coverage and connectivity of the network.

b) The energy consumption trend of the LEACH node and improved LEACH node

The total energy of the simulation is $53.75 \mathrm{~J}$ including 85 ordinary nodes $0.5 \mathrm{~J}$ per one, and 15 high energy nodes, $0.75 \mathrm{~J}$ per one. Statistics death situation of 100 nodes in round $\begin{array}{lllllllll}1100 & 1200 & 1300 & 1400 & 1500 & 1600 & 1700 & 1800,1900 \text { as }\end{array}$ listed in Table III .

TABLE III. LAECH ALGORITHM AND IMPROVED LAECH ALGORITHM STATISTICS ON ENERGY CONSUMPTION

\begin{tabular}{|c|c|c|c|c|c|c|c|c|c|}
\hline Simulation round & 1100 & 1200 & 1300 & 1400 & 1500 & 1600 & 1700 & 1800 & 1900 \\
\hline $\begin{array}{l}\text { The number of } \mathrm{LEACH} \\
\text { algorithm s dead nodes }\end{array}$ & 6 & 7 & 18 & 38 & 75 & 92 & 94 & 95 & 97 \\
\hline $\begin{array}{l}\text { LEACH algorithm for energy } \\
\text { consumption/J }\end{array}$ & 3 & 3.5 & 9 & 19 & 37.5 & 47.75 & 49.25 & 50 & 51.5 \\
\hline $\begin{array}{l}\text { The number of improved } \\
\text { LEACH algorithm s dead nodes }\end{array}$ & 1 & 3 & 4 & 5 & 11 & 55 & 76 & 92 & 96 \\
\hline $\begin{array}{l}\text { Improved LEACH algorithm for } \\
\text { energy consumption/J }\end{array}$ & 0.75 & 2.25 & 3 & 3.75 & 9.25 & 30 & 42.75 & 49.5 & 51.5 \\
\hline
\end{tabular}

As can be seen from the table 3, the energy consumption of the improved LEACH algorithm is less than LEACH algorithm before 1500 rounds. And in the process of the experimental simulation, previous 7 dead nodes are high energy, remaining 1 node after 1700 rounds. The energy consumption of the improved LEACH algorithm is less than LEACH algorithm before 1800 rounds.

Experimental results show that the design of improved LEACH algorithm has practical significance and is important to decrease the energy consumption of the entire network. The improved LEACH algorithm in the monitoring area can let node with larger residua energy be the cluster head node first, to reduce the pressure the of monitoring node with smaller residual energy, balancing of the whole network energy consumption, which make the entire network stabilized state, protecting the node with small residual energy, and it is important to extend the life cycle of the network. As Figure.4.

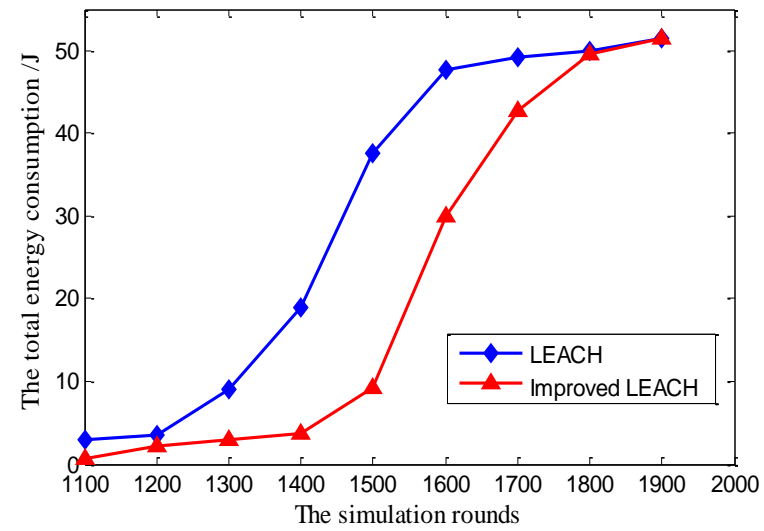

Figure 4. The total energy consumption trend of LEACH algorithm node and improved LEACH algorithm node
Due to LEACH algorithm s problems affect the ray wireless monitoring interface $\mathrm{s}$ performance, an improved LEACH algorithm has been raised. Experimental results show that the improved LEACH algorithm improved LEACH algorithm for cluster heads selection, delay the first nodes death cycle obviously, prolong the life cycle of the entire network, and decrease the energy consumption of the entire network of nodes. The improved algorithm significantly reduced the mortality rate node, guaranteed the network's coverage and connectivity. It has advantageous to the balance utilization of energy. Finally we compared the improved algorithm with LEACH algorithm. The conclusion was that the improved algorithm still has the advantage in terms of stability. It not only prolonged the life cycle of the entire network but also reduced the mortality rate of the whole network energy consumption and guaranteed the performance of the entire network at the same time, which made the nodes tend to focus on death.

\section{ACKNOWLEDGMENTS}

This Research was supported by the National Natural Science Foundation of China under Grant 61403183, key Project of the Hunan Provincial Education Office Science Research of China under Grant 14A121, the Hunan Provincial Natural Science Foundation of China under Grant 2015JJ6096, the Construct Program of the key Laboratory in University of South China (computer science and technology).

\section{REFERENCES}

[1] Zhen Wu and XinYu Jin. The improvement of LEACH in Wireless Sensor Net Works". Chinese Journal of Sencors and actuators, vol. 9, no. 1, Jan. 2006, pp. 1005-9490. 
[2] Yu Zhang. Cluster head distance HAD-LEACH algorithm in WSN, Computer Engineering and Applications, vol. 43(20), 2007, pp.124127.

[3] C. M. Liu and C. H. Lee, Power efficient communication protocols for data gathering on mobile sensor networks, in Proceedings of the 60th IEEE Vehicular Technology Conference (VTC 04), pp. 4635 4639, September 2004

[4] GuoAn Zhao. The Internet of things/sensor experimental teaching materials. BeiJing: Science press, 2011.

[5] LiLin Wang. Ultra low power wireless sensor network node system research and implementation method. HuNan:Hu Nan Univercity, 2009.

[6] R. C. Shah and J. M. Rabaey, Energy aware routing for low energy ad hoc sensor network, Proc IEEE Wirwless Communications and Networking Conferenee (WCNC 02), IEEE, 2002, vol. 1, no. 3, pp. $17-21$
[7] M. Ye, C. F. Li and G. H. Chen, EECS:An energy efficient clustering scheme in wireless sensor networks. IEEE, 2005, pp. 535-540

[8] A. Perrig, R. Szewezyk, SPINS: Security protocols for sensor networks, Wireless Networks, vol. 8, no. 5, 2002, pp. 521-534.

[9] Kan Baoqiang, Cai Li, Zhu Hongsong. Accurate energy model for WSN node and its optimal design, Science Direct, vol. 19, no.3, 2008, pp. $427-433$

[10] M. B. Yassein, A. Al-zou bi. Y. Khamayseh, and W. Mardini, Improvement on LEACH protocol of wireless sensor network, Journal of Digital ontent Technology and Its Applications, vol. 3, no. 2, pp. 260264 ,

[11] G.Ran,H.Zhang,andS.Gong, Improving on LEACH protocol of wireless sensor networks using fuzzy logic, Journal ofInformation and Computational Science, vol. 7, no. 3, pp. 767 775, 2010.

[12] Hao Pan QinFen Dong.. Wireless sensor network operating system TinyOS. Beijing: tsinghua university press 2011 